Culture et histoire dans l'espace roman

$12 \mid 2014$

Empreintes/emprunts : entre forces de conformisation et forces d'innovation

\title{
Le narrateur dans El Conde Lucanor : une voix empruntée, un double « je »?
}

\section{MARTA LACOMBA}

\section{(2) OpenEdition}

\section{Journals}

Édition électronique

URL : https://journals.openedition.org/cher/7200

DOI : $10.4000 /$ cher.7200

ISSN : 2803-5992

Éditeur

Presses universitaires de Strasbourg

Édition imprimée

Date de publication : 7 juillet 2014

Pagination : 235-254

ISBN : 978-2-86820-569-8

ISSN : 1968-035X

Référence électronique

MARTA LACOMBA, « Le narrateur dans El Conde Lucanor : une voix empruntée, un double « je »? », reCHERches [En ligne], 12 | 2014, mis en ligne le 13 décembre 2021, consulté le 15 décembre 2021. URL : http://journals.openedition.org/cher/7200 ; DOI : https://doi.org/10.4000/cher.7200

\section{(c) (i) (8)}

Ce(tte) œuvre est mise à disposition selon les termes de la Licence Creative Commons Attribution -

Pas d'Utilisation Commerciale - Partage dans les Mêmes Conditions 4.0 International. 


\title{
Le narrateur dans El Conde Lucanor: une voix empruntée, un double "je»?
}

\author{
MARTA LACOMBA \\ Université de Bordeaux 3
}

$E_{\text {l'infant don Juan Manuel, neveu d'Alphonse X et l'un des personnages }}^{\text {Libro de los enxiemplos del Conde Lucanor et de Patronio est l'œuvre de }}$ capitaux de la cour dans la première moitié du XIve siècle. El Conde Lucanor est un recueil de contes destinés, d'après les deux prologues de l'œuvre, à fournir un savoir didactique.

Le premier lien évident entre ce livre et la thématique autour de laquelle se réunissent ces actes de colloque, empreintes et emprunts, tient au fait, simple, évident mais incontournable, que cette œuvre a laissé une trace, une empreinte dans la littérature. Elle fut rédigée entre 1330 et 1335 et nous nous en occupons encore maintenant, en 2012. Son influence, son empreinte, peut également être mesurée à l'aune du nombre de manuscrits qui sont parvenus jusqu'à nous et qui sont au nombre de six, un chiffre supérieur à celui de toute autre œuvre médiévale castillane. À titre de comparaison, il convient de rappeler qu'il n'existe qu'un seul manuscrit du Cantar de mio Cid - qui de plus est incomplet - et trois du Libro de Buen Amor. Pour ce qui est des emprunts, de l'héritage, El Conde Lucanor s'inscrit dans une tradition littéraire, génériquement constituée autour de la transmission d'exempla, à laquelle ce livre n'est pourtant pas réductible, comme nous essaierons de le montrer ici.

Ce n'est pas dans une perspective diachronique, ni en amont ni en aval, que nous entendons aborder ici cette œuvre. Si El Conde Lucanor a laissé une trace, cette empreinte est en grande partie due aux innovations que don Juan Manuel apporte. Ce dernier est en effet considéré comme le premier

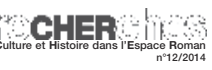


écrivain à avoir eu une conscience d'auteur, ou en tout cas à l'avoir exprimée de façon explicite. C'est notamment dans les prologues aux différentes parties de l'œuvre qu'apparaît l'empreinte de l'auteur, de ce personnage qui, nous le verrons, se situe à la fois à l'extérieur et à l'intérieur du récit. Dans cette affirmation de la puissance de son pouvoir créateur, la figure de l'auteur octroie également une large marge de manœuvre à la figure du narrateur. Notre hypothèse est que c'est précisément à travers les empreintes ostensibles et revendiquées que ces deux figures laissent sur la «scène du crime» que se produit le renouvellement de l'espace littéraire. Ce sont donc ces différentes traces, laissées aussi bien par l'auteur que par le narrateur, que nous tenterons d'identifier ici, dans le but de montrer que ce jeu de pistes ouvre la voie d'un nouveau cadre d'écriture.

Dans un premier temps seront ainsi examinés les emprunts vis-à-vis de la tradition, de façon à en mesurer l'assimilation ou l'écart, non pas du point du vue de la matière héritée mais du cadre de la narration. La deuxième partie de ce travail sera consacrée aux marques, aux empreintes qu'auteur et narrateurs laissent, exposent, construisent tout au long de ces prologues. Il s'agira ensuite de proposer une interprétation à ce jeu d'empreintes et d'emprunts.

\section{L'héritage}

La thématique de l'empreinte et de l'emprunt est, dans le contexte médiéval, indissociable des notions de création et d'assimilation, de rupture et de continuité. C'est donc dans cette perspective que nous avons tout d'abord orienté notre réflexion, ce qui nous a forcément conduit vers deux notions complémentaires, la réécriture et le remploi. Dans un article où elle explore avec fécondité ces deux pratiques médiévales à travers le cadre théorique conçu par la narratologie, Monique Goullet (Goullet 2006 : 21) établit que réécriture et remploi relèvent de deux fonctionnements opposés. Alors que la réécriture fait du neuf avec du vieux, le remploi est l'insertion du vieux dans du neuf. Alors que le remploi est une technique, comme pourrait l'être l'emprunt, la réécriture est une pratique. Et dans cette pratique, ou à travers cette pratique, l'affichage de la continuité masque le plus souvent l'empreinte de la rupture. Ce sont les continuités, sinon affichées du moins héritées, qui seront ici examinées, de façon à mesurer ensuite l'empreinte de ces cadres sur le canevas proposé par don Juan Manuel. 
El Conde Lucanor peut être considéré comme un recueil d'exemples, d'enseignements que le conseiller Patronio livre à son seigneur, le comte Lucanor. Il s'agit donc d'une œuvre didactique structurée par le dialogue. Il ne convient pas de s'attarder ici sur les origines, la diffusion, le contenu ni la tradition de ce genre littéraire. Des études comme celles de Gimeno Casalduero se sont intéressées aux différentes méthodes, exemples, sentences, réflexion théorique, utilisées pour aborder cette matière didactique (Gimeno Casalduero 1977: 19-20). Or c'est le cadre de narration que le recours aux exempla véhicule qu'il nous importe de rendre explicite. Ce genre d'ouvrages utilise la technique de la compilation et du remploi, et même si les matériaux récupérés, empruntés, sont bien évidemment recyclés et réécrits, ils sont généralement présentés comme si la seule opération à laquelle ils avaient éte soumis était celle de la transmission. La réécriture, tout comme la traduction d'ailleurs, est souvent assimilée à de simples opérations de déplacement, d'un texte à un autre, ou d'une langue à une autre. Or il est bien évident que ce rôle de simple passeur que s'attribuent la traduction et la compilation médiévales ne saurait être qu'un leurre dont personne n'est, nous semblet-il, dupe. Autrement dit, il s'agit d'une topique, d'un schéma explicatif qui structure les liens tant entre le monde référentiel et l'œuvre, qu'entre l'œuvre et les œuvres la précédant, mais encore entre auteur et destinataires. Une topique qui prétend que ces liens sont marqués par la spécularité, comme nous l'avons tenté de le montrer ailleurs (Lacomba 2011: 120-129). Dans le cas des exempla, mais pas seulement car il s'agit d'une topique très largement étendue, tout se joue dans l'antériorité et l'extériorité. Le compilateur ne ferait que reprendre des exempla antérieurs - et donc extérieurs - à sa propre œuvre, ce qui est plutôt vrai du point de vue du contenu, en grande partie inexact en ce qui concerne la forme et totalement faux pour ce qui est de l'essentiel, c'est-à-dire le sens de la nouvelle compilation. Mais, dans cette topique, dans ce paradigme littéraire, la matière transmise est considérée comme étant à la fois antérieure et extérieure au compilateur, dont l'œuvre ne serait qu'une sorte de miroir. Cette prétendue spécularité serait double, car la matière des exempla prétend puiser dans le vécu, dans des histoires vraies qui seraient arrivées à de vraies personnes. Autrement dit, tout se passe comme si produire des recueils d'exempla n'était affaire que de remploi ou d'emprunt. Ceux qui auraient mis par écrit (ou raconté) en premier ces histoires n'auraient fait que donner une forme verbale à de vrais faits et les compilateurs successifs ne feraient donc que transmettre un savoir préexistant. La topique de la spécularité repose donc, dans une grande mesure, sur le fait de prétendre faire passer la création pour une 
simple technique. Il convient ici de bien insister sur le fait qu'il s'agit bien de prétendre, de faire comme si. Mais lorsque tout le monde fait comme si, ce comme si devient un paradigme littéraire.

Le deuxième héritage qui pourrait être associé à l'œuvre de don Juan Manuel, et le mot héritage est ici doublement pertinent, est celui des textes produits par son oncle, le roi Alphonse X (1252-1284). Ce dernier est responsable d'une série d'ouvrages à contenu historique, juridique et scientifique qui, d'après nos critères littéraires et donc anachroniques pour le XIII ${ }^{e}$ siècle, n'appartiendraient pas à des genres fictionnels. Le terme littérature serait même non pertinent pour la période médiévale, comme le souligne Funes, qui place au centre de sa réflexion les spécificités historiques et discursives des œuvres (Funes 2009: 15-55). Or, dans toutes ces œuvres, notamment à travers les prologues mais pas seulement, le roi Alphonse brise précisément cette topique de la spécularité en mettant en avant sa volonté et son action, celles de l'auteur, et ce dans toutes les étapes du processus de création. Comme le dit Leonardo Funes (Funes 1997: 72): «En suma, el exhaustivo conocimiento del pasado que la ciencia provee y la razón ordena se proyecta hacia el público por la voluntad didáctica del autor, realizada en el contexto de enunciación que la prosa alfonsí pone de manifiesto».

La compilation et la traduction, tout autant que le choix des sources et le but de l'œuvre dépendent, dans ce nouveau paradigme, de l'auteur-acteur. Dans les prologues des œuvres scientifiques, par exemple, c'est le roi luimême qui serait à l'origine de la récupération matérielle du texte proposé - mais il s'agit, bien entendu, là encore d'une topique, d'un comme si accepté de tous. Cela est notamment marqué dans le prologue du Lapidario (Alphonse X: 17).

Par ailleurs, toutes les œuvres alphonsines reposent sur l'idée d'une accumulation raisonnée de la matière ordonnée selon des critères explicites, dans le but de transmettre un savoir qui permettra à ses sujets de mieux agir, comme le souligne Gómez Redondo à plusieurs reprises (par exemple, Gómez Redondo 1998, t.1: 405 et 417). Le roi-auteur est présent de plusieurs façons et à tous les moments de la création. Il est en même temps à l'origine du projet, au cœur du processus et des opérations qui permettent la réalisation concrète de l'œuvre, mais il se place également face au livre comme résultat, en rendant explicites les raisons qui déterminent l'écriture. Le roi-acteur n'est pas extérieur au récit, bien au contraire, il intervient à chacune de ces étapes. Le livre ne reproduit, ne réfléchit donc pas l'ordre du monde, il l'infléchit en le rendant conforme à l'ordre royal. Si la topique 
de la spécularité, héritage platonico-augustinien, pose la transparence du passeur, le paradigme alphonsin fait de l'auteur l'agent, la cause efficiente, pour reprendre les termes de la pensée aristotélicienne, de la transformation du monde (Lacomba 2011: 25-29).

Il s'agira maintenant de montrer que, si El Conde Lucanor hérite, bien évidemment, la matière exemplaire, cette dernière est cependant ici libérée de la topique qui la configurait. Ce n'est pas pour autant la voie ni la voix alphonsine qu'emprunte don Juan Manuel.

\section{Narrateurs et auteur}

L'œuvre compte deux prologues. Le premier est porté par un narrateur qui parle de l'auteur, don Juan Manuel, à la troisième personne. Dans le deuxième, c'est don Juan Manuel, l'auteur du livre, qui parle à la première personne.

Dans le premier prologue, le narrateur omniscient connaît les motivations et les objectifs de l'auteur: "Et puso en el [libro] los enxiemplos más aprovechosos que el sopo de las cosas que acaescieron porque los omnes puedan fazer esto que dicho es" in El Conde Lucanor, éd. Blecua, désormais $E C L,(E C L: 45)$. Ce narrateur semble donc affirmer un double rapport de spécularité entre le monde et le livre. Un rapport qui s'exprime en termes d'antériorité, dans la mesure où le livre recueille des « choses qui seraient arrivées ", mais également en termes de postériorité, puisque le livre doit permettre aux lecteurs de mieux agir dans le monde. Il s'agit donc d'un double mouvement qui est ici revendiqué, du monde vers le livre, dans un premier temps, puis du livre vers le monde.

Cependant, le fait même que ce discours soit placé dans la bouche d'un narrateur déconstruit le paradigme qu'il est censé revendiquer. Le cadre de la spécularité est contenu dans un autre cadre. N'étant pas contenant, mais contenu, il se trouve par là-même vidé de sa valeur cadrante, de son pouvoir de contenir, de proposer une interprétation pour tout. La spécularité n'est donc plus l'ensemble, mais un élément d'un nouvel ensemble dont il convient de définir les caractéristiques.

Ainsi, il faut tout d'abord signaler que le nouveau cadre ici proposé fait intervenir un narrateur omniscient qui, se plaçant à l'extérieur de l'œuvre, évoque un auteur qui est, effectivement, un des grands acteurs de la vie politique castillane du XIVe siècle. Or c'est une voix sans existence dans le monde réel qui va être chargée de présenter l'œuvre de l'infant de Castille. 
Le personnage va parler à la place de la personne. Ce jeu dans lequel don Juan Manuel porte d'abord le masque du narrateur, d'un narrateur dont la matière est en fait l'œuvre de ce même Juan Manuel masqué, pose d'emblée le décalage que El Conde Lucanor imprime tant vis-à-vis de la topique de la spécularité que du dirigisme autorial d'Alphonse X. Pour mettre en évidence ce jeu de miroirs, étudié dans un cadre plus large par Avalle Arce (Avalle Arce 2006: 56-64), il convient de mettre en parallèle les deux prologues de l'œuvre. Voici le début du premier prologue:

Este libro fizo don Iohan, fijo del muy noble infante don Manuel, deseando que los omnes fiziessen en este mundo tales obras que les fuessen aprovechosas [...]. Et puso en él los enxiemplos más aprovechosos que él sopo de las cosas que acaesçieron porque los omnes puedan fazer esto que dicho es (ECL: 45).

La première chose à signaler dans ce premier prologue est que l'énoncé que prend en charge le narrateur inclut en fait l'auteur. Examinons maintenant le deuxième prologue, dans lequel don Juan Manuel s'exprime à la première personne :

[...] Por ende, yo, don Johan, fijo del infante don Manuel, adelantado mayor de la frontera et del regno de Murçia, fiz este libro compuesto de las más apuestas palabras que yo pude [...]. Et esto fiz segund la manera que fazen los físicos, que quando quieren fazer alguna melizina que aproveche al fígado, por razón que naturalmente el figado se paga. Et pues el prólogo es acabado, de aquí en adelante començaré la manera del libro, en manera de una grand señor que fablava con un su consegero. Et dizían al señor conde Lucanor, et al consegero, Patronio (ECL: 50-52).

Lorsque, dans ce deuxième prologue, l'auteur prend la parole, il apparaît que cela répond, correspond à ce que le narrateur avait en effet annoncé dans le premier prologue. Tout se passe comme si les rôles du narrateur et de l'auteur avait été inversés, celui-ci étant chargé de l'énoncé, celui-là de l'énonciation.

Il faut signaler qu'un deuxième élément vient brouiller les pistes : c'est le fait que l'auteur, ou ce personnage qui parle au nom et avec le nom de l'auteur, a choisi le mode dialogal comme manière de faire passer son enseignement, sa melizina. Cela veut dire qu'il semble établir une distinction entre contenu et contenant, entre finalité et moyens. Mais dès le premier exemple, dès que l'on quitte la matière prologale, il apparaît que le dialogue annoncé et encadré donc par l'auteur, est en fait assumé par une nouvelle figure de narrateur omniscient. Ce dernier peut en effet rendre compte de ce que se disent en absence de tout témoin Patronio et le comte Lucanor : 
" acaescio una vez que el conde Lucanor estaba fablando en su poridat con Patronio, su consegero, et dixol [...]» (ECL: 52).

Cette deuxième figure de narrateur ne pourrait en aucun cas se confondre avec celle de la personne qui s'exprime dans le deuxième prologue car celle-ci avait bien pris soin de marquer qu'elle prenait congé du lecteur en lui faisant part de la manière dont serait à partir de là construit son récit. La coupure par rapport au prologue marque le début de ce dernier. Ce deuxième narrateur appartient donc bien à l'énoncé, à l'histoire racontée. En guise de récapitulation, rappelons donc que, d'un côté, la figure de l'auteur est encadrée par le discours du narrateur du premier prologue, mais, d'un autre côté, sert de cadre au discours du narrateur du premier exemple.

Autrement dit, le lien entre le narrateur (n) du premier exemple et l'auteur (JM, pour Juan Manuel) qui parle dans le deuxième prologue est de même nature que celui qui s'établit entre ce dernier et le narrateur du premier prologue $(\mathrm{N})$. Ce double rapport peut être exprimé de la façon suivante: $\mathrm{N} \rightarrow[\mathrm{JM} \rightarrow(\mathrm{n})]$

Cette modélisation permet de mettre en évidence non seulement le mécanisme d'enchâssement, mais également le fait que l'auteur, JM, est pris dans un cadre (représenté par les parenthèses) tout comme n, le narrateur du premier exemple. Cela fait donc apparaître, de façon explicite, le caractère intradiégétique et donc fictionnel de la figure du don Juan Manuel qui parle dans le deuxième prologue.

Ce jeu de dédoublement de l'auteur, ce jeu du «je», qui est à la fois auteur et personnage, se confirme et s'amplifie à la fin du premier exemple: «Et entendiendo don Iohan que estos enxiemplos eran muy buenos, fizolos escribir en este libro, et fizo estos viessos en que se pone la sentencia de los enxiemplo" (ECL: 60).

La question qui se pose ici serait: mais qui est donc ce don Juan Manuel? Car il apparaît que le récit ne fait pas ici ce que le cadre de l'énonciation semblait lui imposer. On assiste ainsi à une rupture du cadre narratif à travers le dédoublement d'un auteur/personnage nommé don Juan Manuel. Le premier don Juan Manuel, celui que le narrateur du premier prologue présente à la troisième personne comme étant l'auteur d'une œuvre didactique, serait en effet l'écrivain, qui s'exprime à la première personne dans le deuxième prologue et pose le cadre de l'œuvre à venir. Ce Juan Manuel écrivain semble s'effacer avant que le récit ne commence. 
Le deuxième don Juan Manuel, celui du premier exemple, apparaît à nouveau à la troisième personne puisqu'il est introduit par le narrateur omniscient portant le récit depuis la fin du deuxième prologue, qui devait en toute logique impliquer l'effacement de l'écrivain, de même que la fin $\mathrm{du}$ premier prologue marque la disparition du premier narrateur. Or ce deuxième don Juan Manuel connaît parfaitement la matière racontée par ce deuxième narrateur et se l'approprie comme si elle lui était extérieure. Le trompe l'œil ici mis en place fait en effet passer le récit pour l'œuvre conjointe du deuxième narrateur et de ce troisième don Juan Manuel. Ce dernier serait l'auteur des distiques («fizo estos viessos», ECL: 60) qui contiennent l'enseignement de chaque récit mais ne ferait que transcrire les exempla ( do don Iohan que estos enxiemplos eran muy buenos, fizolos escribir en este libro", ECL: 60). Et surtout, le don Juan Manuel qui est l'auteur de ces vers devient à son tour le personnage à la fois du narrateur du récit et du don Juan Manuel du deuxième prologue. En reprenant le modèle précédant, la structure de cette nouvelle mise en abîme pourrait s'exprimer de la façon suivante :

$$
\mathrm{N} 1 \rightarrow[J M 1 \rightarrow(\text { n2 } \underset{\text { JMM } 2)}{\rightarrow \text { Patronio/ Lucanor })]}
$$

Toute la première partie de l'œuvre repose donc sur cette construction en trompe-l'œil qui établit d'étranges relations entre narrateurs et auteur.

Or le prologue de la deuxième partie - elle aussi consacrée à des exemples mais de nature plus obscure - vient également rendre plus obscur, mais à la fois peut-être plus clair le cadre énonciatif. Le premier élément à signaler est l'apparition d'un troisième don Juan Manuel. Celui-ci s'exprime à la première personne et pourrait en fait être assimilé au premier don Juan Manuel, celui qui apparaît comme étant l'auteur de l'œuvre: «Después que yo, don Iohan [...] ove acabado este libro del conde Lucanor et de Patronio [...]» (ECL: 277). Ce Juan Manuel vient en fait déconstruire l'artefact narratif de la première partie, puisqu'il fait savoir qu'il est l'auteur, non seulement des vers, comme l'affirmait le deuxième don Juan Manuel, mais, comme l'affirmait également le premier don Juan Manuel, de l'ensemble du récit. Autrement dit, l'auteur montre ici que c'est bien lui qui impose et qui crée toutes ces figures de narrateurs et tous ces doubles, tous ces mirages de la figure d'un auteur tout-puissant. Car c'est bel et bien son pouvoir créateur, son art de tromper, de feindre, de construire une fiction qui est constamment exercé. Ainsi, alors même que ce dernier don Juan Manuel vient de montrer les rouages de la machine, en faisant apparaître que le 
deuxième don Juan Manuel n'était qu'un personnage, par un nouveau tour de force, il la fait repartir à nouveau. Ce nouveau prologue se termine en effet comme celui de la première partie : «et la manera del libro es que Patronio fabla con el conde Lucanor" (ECL: 278). Cela implique de céder à nouveau la parole au narrateur omniscient, qui reprend en effet le rôle de témoin des conversations entre comte et conseiller, comme le montre le recours au discours direct et aux verbes dicendi: "Señor conde Lucanor - dixo Patronio» (ECL: 278). Ce «dixo Patronio» ne peut être bien évidemment soutenu que par un narrateur omniscient. Or, et c'est là le nouvel artifice de l'auteur, la voix de ce narrateur entre ici en conflit avec celle de Patronio, car ce dernier revendique la responsabilité non seulement des conseils qu'il offre à son seigneur mais également du récit. Patronio prend en charge non seulement l'ensemble des exempla déjà racontés mais également ceux à venir. Il affirme: "Yo vos fablé fasta agora lo más declaradamente que yo pude, e [...] fablarvos he daquí en adelante essa misma manera, más non en essa manera que en l'otro libro antes deste» (ECL: 278-279). Et c'est bien le rôle de l'auteur que Patronio endosse car il n'assume pas seulement le récit mais également le livre.

Le prologue de la troisième partie maintient cette double identification. $\mathrm{Si}$ l'on entend toujours la voix d'un narrateur qui serait témoin et transmetteur de la conversation entre Patronio et Lucanor (comme le montrent les «dixo Patronio» et les «dixo Lucanor»), Patronio est cependant toujours aussi bien celui qui raconte que celui qui est responsable du livre: «después que el otro libro fue acabado [...] comencé a fablar en este libro más avreviado et más oscuro que en l'otro" (ECL: 288).

Le prologue de la quatrième partie renforce ce schéma: Patronio assume maintenant la responsabilité narrative et structurale de tout ce qui a été raconté: «[...] trabajé de vos dezir algunas cosas más de las que vos avía dicho en los enxiemplos que vos dixe en la primera parte deste libro [...]; et pues que en la segunda deste libro [...] (ECL:295).

Il rend également explicite - comme l'avait fait le Juan Manuel auteur dans les prologues de la première et de la deuxième partie - la fin du discours contenant et le début du récit: "[...] daquí adellante parad bien mientes a lo vos diré» (ECL: 296). Le fait que Patronio s'attribue le rôle d'architecte du livre implique que, dans cette mise en scène, le deuxième Juan Manuel et même le troisième, deviennent ses créatures, ses personnages.

Don Juan Manuel, ou plutôt celui qu'il faudrait appeler Juan Manuel 4, apparaît dans le prologue de la cinquième partie pour déconstruire à 
nouveau la récurrence du schéma narratif. Il convient de souligner avant tout que c'est la voix de Patronio qui le fait surgir, à la troisième personne à nouveau: "[...] et pues tantas cosas son escriptas, por talante que don Johan, ovo de complir talante de don Jayme, digo vos que non quiero fablar ya en este libro de enxiemplos [...]» (ECL:300).

La fin de l'œuvre est également révélatrice de ce jeu de dédoublements. Patronio, toujours narrateur à la première personne, entendant être également le sujet de l'énonciation et pas seulement de l'énoncé, annonce: «Agora, señor conde Lucanor, [...] vos he dicho assaz [...], tengo que vos he compido acabado todo lo que vos dije. Et pues assí es, en esto fago fin a este libro.Et acabolo don Iohan en Salmerón, lunes XII día de junio, era de mil et CCC et LXX et tres años» (ECL: 321-322).

En guise de conclusion, il convient de souligner que, tout au long de cette œuvre, se met en place un jeu de doubles, dont le fonctionnement est désarticulé à plusieurs reprises et à plusieurs reprises remis en marche. En se démasquant, l'auteur est loin d'annuler l'effet des ses artifices, bien au contraire, il en décuple la puissance. Il devient ainsi, comme dans une sorte de retable de Maese Pedro avant l'heure, un marionnettiste dont l'une des marionettes serait Don Juan Manuel lui même. El conde Lucanor adopte donc, par rapport aux deux paradigmes littéraires que cette œuvre aurait pu emprunter (celui de la spécularité ou celui d'un auteur ordonnateur d'un récit linéaire et exhaustif), sa propre voie, sa propre voix, celle d'un auteur qui crée ses propres miroirs.

Certes, El conde Lucanor est une œuvre didactique mais, tout comme Les Mille et Une Nuits, ne saurait être définie par cette adscription d'ordre générique ni par son contenu. Nous avons essayé ici de mettre en évidence la complexité et la subtilité de son contenant, qui ouvre la voie, par ce double «je», par ce jeu de doubles, à l'affirmation de la puissance et du pouvoir de la fiction, des fictions, des Ficciones...

\section{Bibliographie}

Avalle Arce, J. B., 2006, Las novelas y sus narradores, Madrid, Biblioteca de Estudios Cervantinos, Ediciones del Centro de Estudios Cervantinos.

Funes, L., 1997, El modelo historiográfico alfonsí: una caracterización, Londres, Queen Mary and Westfield College, (Papers of the Medieval Hispanic Research Seminar,6). Funes, L., 2009, Investigación literaria de textos medievales: objeto y práctica, Buenos Aires, Miño y Dávila, p. 11-55. 
Gimeno Casalduero, J., 1977, La creación literaria de la Edad Media y del Renacimiento, Madrid, Porrúa Turanzas.

Goullet, M., 2006, «Reutilización, actualización: quelques réflexions préliminaires», Réécriture et falsification dans l'Espagne médiévale, Cahiers d'études hispaniques médiévales $\mathrm{n}^{\circ} 29$, p. 11-21.

Juan Manuel, 1992, El Conde Lucanor, éd. J. M. Blecua, Madrid, Clásicos Castalia,

Lacomba, M., 2011, "Les prologues scientifiques alphonsins et la symbolique du miroir », in Le Miroir du prince. Écriture, transmission et réception dans l'Europe méridionale (XIII ${ }^{\mathrm{e}}-\mathrm{XIV}^{\mathrm{e}}$ siècle), Bordeaux, Presses Universitaires de Bordeaux, p. 113-129.

Gómez Redondo, F., 1998, Historia de la prosa medieval castellana, vol. I: La creación del discurso prosístico: el entramado cortesano, Madrid, Cátedra.

Rodríguez Montalvo, S. et Lapesa R. (éd) "Lapidario": (según el manuscrito escurialense H.I. 15), 1981, Madrid, Gredos (Biblioteca Románica Hispánica, IV: Textos, 14). 